\title{
Optimization of Scan Time in MRI for Total Hip Prostheses: SEMAC Tailoring for Prosthetic Implants Containing Different Types of Metals
}

\section{Optimierung der MRT-Messzeit bei Totalendoprothesen: SEMAC zugeschnitten auf Implantate aus verschiedenen Metallen}

Authors

Affiliations
X. Deligianni ${ }^{1}, 3$, O. Bieri ${ }^{1}$, R. Elke ${ }^{2}$, T. Wischer ${ }^{3}$, T. Egelhof ${ }^{3}$

Department of Radiology, Division of Radiological Physics, University of Basel Hospital, Basel, Switzerland

Orthomerian, Basel, Switzerland

Institute of Radiology, Merian Iselin Klinik, Basel, Switzerland
Key words

- prostheses

- imaging sequences

- femur received $\quad 3.3 .2015$

accepted 10.7.2015

\section{Bibliography}

DOI http://dx.doi.org/ 10.1055/s-0041-104893

Published online: 1.9.2015

Fortschr Röntgenstr 2015; 187:

1116-1122 @ Georg Thieme

Verlag KG Stuttgart - New York . ISSN 1438-9029

\section{Correspondence}

Frau Dr. Xeni Deligianni Department of Radiology, Division of Radiological Physics, University of Basel Hospital Petersgraben 4

4031 Basel

Switzerland

Tel.: +41615565728

Fax: +41612654348

xeni.deligianni@unibas.ch

\section{Zusammenfassung}

$\nabla$

Einführung: Die Magnetresonanztomografie (MRT) der periprothetischen Weichteile nach Hüftendoprothetik ist von grosser klinischer Relevanz für die Diagnose verschiedenster Erkrankungen, die mit anderen bildgebenden Verfahren nicht darstellbar sind. Aus diesem Grund wird viel Forschungsarbeit in die Entwicklung von MRT-Techniken zur Reduzierung von Metallartefakten investiert. Ein Beispiel hierfür ist SEMAC - eine Methode, die mittels Schichtkodierung dazu beiträgt, metallinduzierte Artefakte abzuschwächen. Grundsätzlich korreliert die Stärke der Artefaktverringerung bei der Anwendung von SEMAC direkt mit der Messzeit. Es ist jedoch bis anhin unklar, wie die Parameter der MRT-Sequenz abhängig vom Material des Implantats am effizientesten eingestellt werden sollten. Das Ziel dieser Arbeit ist es, SEMAC MRT-Protokolle zu optimieren, sodass sie auf die jeweiligen Implantatmaterialien zugeschnitten sind.

Material und Methoden: Fünf der am häufigsten verwendeten Hüftprothesen (1. Revisionsprothese (S-Rom), 2. Titanium-Legierung, 3. Müller-Typ (CoNiCrMo-Legierung), 4. Old Charnley-Prothese (Exeter / Stryker), 5. MS-30-Schaft (Edelstahl) wurden in einem 1,5 Tesla MRT-Scanner mit einer klinischen SEMAC-Sequenz und unterschiedlicher Anzahl an Schichtkodierungsschritten (SES) gemessen (2-23 Schritte). Die Verkleinerung der von Artefakten betroffenen Fläche im Vergleich zur maximalen Artefaktunterdrückung wurde quantitativ und qualitativ ausgewertet mit dem Ziel, für jede der fünf gemessenen Prothesen eine optimale Anzahl an Schichtkodierungsschritten zu finden. Die resultierende Scanzeit variierte von 1 bis $10 \mathrm{~min}$. Ergebnisse: Die Anzahl der SES, die das Artefaktvolumen auf unter $300 \mathrm{~mm}^{3}$ reduzierte, reichte von 3 bis 13 abhängig vom Implantatmaterial. Die Ergebnisse zeigten, dass für Titanprothesen bereits 3 SES ausreichen können, um Metallarte-

\section{Abstract \\ $\nabla$}

Introduction: Magnetic resonance imaging (MRI) of soft tissues after total hip arthroplasty is of clinical interest for the diagnosis of various pathologies that are usually invisible with other imaging modalities. As a result, considerable effort has been put into the development of metal artifact reduction MRI strategies, such as slice encoding for metal artifact correction (SEMAC). Generally, the degree of metal artifact reduction with SEMAC directly relates to the overall time spent for acquisition, but there is no specific consensus about the most efficient sequence setup depending on the implant material. The aim of this article is to suggest material-tailored SEMAC protocol settings.

Materials and Methods: Five of the most common total hip prostheses (1. Revision prosthesis (SRom), 2. Titanium alloy, 3. Müller type (CoNiCRMo alloy), 4. Old Charnley prosthesis (Exeter/ Stryker), 5. MS-30 stem (stainless-steel)) were scanned on a 1.5 T MRI clinical scanner with a SEMAC sequence with a range of artifact-resolving slice encoding steps (SES: 2-23) along the slice direction (yielding a total variable scan time ranging from 1 to $10 \mathrm{~min}$ ). The reduction of the artifact volume in comparison with maximal artifact suppression was evaluated both quantitatively and qualitatively in order to establish a recommended number of steps for each case.

Results: The number of SES that reduced the artifact volume below approximately $300 \mathrm{~mm}^{3}$ ranged from 3 to 13, depending on the material. Our results showed that although 3 SES steps can be sufficient for artifact reduction for titanium prostheses, at least 11 SES should be used for prostheses made of materials such as certain alloys of stainless steel.

Conclusion: Tailoring SES to the implant material and to the desired degree of metal artifact reduction represents a simple tool for workflow opti- 
fakte effektiv zu reduzieren, während für Prothesen aus anderen Materialien wie Legierungen aus rostfreiem Stahl mindestens 11 SES verwendet werden sollten.

Schlussfolgerung: Das Abstimmen der SES auf das verwendete Implantatmaterial und auf den gewünschten Grad an Artefaktunterdrückung ist ein einfaches Mittel, um die klinische SEMACBildgebung bei Vorliegen einer Totalendoprothese des Hüftgelenks zu optimieren.

Kernaussagen:

- In vitro-Untersuchung der fünf gebräuchlichsten Totalendoprothesen für das Hüftgelenk.

- Bestimmung optimaler SEMAC-Protokolle hinsichtlich der Messzeit.

- Die Optimierungen der T1-gewichteten und der „Inversion Recovery“ SEMAC MRT-Protokolle waren vergleichbar.

- Die vorgeschlagene Anpassung der SEMAC-Protokolle abhängig von den verwendeten Prothesen führt zu kürzeren klinischen Messzeiten. mization of SEMAC imaging near total hip arthroplasty in a clinical setting.

Key Points:

- Five of the most common total hip prostheses have been investigated in vitro.

- Tailored SEMAC protocols - in terms of scan duration - have been determined.

- Tailoring was similar for $\mathrm{T}_{1 \text {-weighted }}$ and inversion recovery SEMAC MRI.

- The suggested prosthesis-related SEMAC adaptation shortens clinical scan times.

Citation Format:

- Deligianni X, Bieri O, Elke R et al. Optimization of Scan Time in MRI for Total Hip Prostheses: SEMAC Tailoring for Prosthetic Implants Containing Different Types of Metals. Fortschr Röntgenstr 2015; 187: 1116-1122

\section{Introduction}

$\nabla$

Total hip arthroplasty (THA) is a common treatment for osteoarthritis [1]. Some of the issues occurring in patients with total hip prostheses (THP) are osteolysis, dislocation, granulomatous disease, loosening and infection. Nowadays, it is feasible to use magnetic resonance imaging (MRI) to investigate these issues [2]. MRI offers the advantage of soft tissue contrast, but it is subjected to magnetic susceptibility artifacts in the presence of metal [3]. Conventional MRI sequences can be optimized to reduce susceptibility-related image artifacts [4] and allow hip imaging even in the presence of metal prostheses, but are still subjected to considerable distortions, such as "pile-up" artifacts and signal voids $[2,5]$. Recently, however, novel MRI sequences have been introduced, such as view angle tilting (VAT) [6], multiple acquisition with variable resonance image combination (MAVRIC) [7], single-point imaging [8] and slice encoding for metal artifact correction (SEMAC) sequences [9] that overcome some of these limitations [2]. VAT is used to correct for in-plane geometric distortions by repeating the slice selection gradient during the signal readout, but suffers from geometric through-plane distortion and blurring effects. In contrast, SEMAC is a multi-slice 2 D TSE sequence that uses both VAT and additional variable slice encoding steps (SES) to correct through-plane artifacts and improve the visualization of anatomical details [10]. Various studies have been performed for the evaluation of SEMAC on reducing susceptibility artifacts in phantoms and in patients [10-15]. Objectively and consistently comparing the efficiency of artifact reduction is an exacting process, since the metal-related artifact form and type can be very irregular [10, 14-16].

Generally, SEMAC offers a considerable reduction of metal-related image artifacts, but requires a considerable increase in scan time - typically diametral to clinical workflow optimizations. Whereas, in principle, a huge number of SES may completely resolve the material-related artifacts issue, the resulting scan time requirements will not be in line with clinical demands. Consequently, a simple tradeoff between prohibitively long scan times and clinically useful images is to use the highest possible SES depending on the predetermined available scan time within the scan protocol. However, in practice there is a wide range of prostheses and materials related to how recent the prosthesis model is. The overall requisite scan time is highly dependent on the type of implant that is present and on the desired degree of image artifact reduction. Depending on the prosthesis material, a larger or smaller number of SES would be sufficient for SEMAC metal artifact reduction. Therefore, several studies have recently taken into account the influence of the implant material on the efficiency of different artifact suppression methods: a) SE, GRE and high bandwidth TSE [14, 17], b) MAVRIC [16] and c) SEMAC [13, 14]. Månson et al. highlighted that a proper number of SES is critical for the performance of SEMAC and also that the material of a prosthesis is a decisive parameter affecting the performance of an artifact reduction technique [13]. However, how the efficiency of the sequence changes for a continuous range of SES and whether it changes for different types of image contrast $\left(T_{1-w}\right.$ versus fat suppression) are not investigated. In this study, we evaluated residual metal-related image artifacts for a $\mathrm{T}_{1-\mathrm{w}}$ and a short-tau inversion recovery (STIR) SEMAC protocol on five of the most common total hip prostheses ( $\bullet$ Fig. 1 ) as a function of the number of SES and thus of the total scan time. The least distorted image, which was acquired with a very high number of SES, was used as a control reference. The aim of this evaluation was to propose a guideline for the minimum number of SEMAC SES steps required to provide adequate, i.e. diagnostically relevant, image quality.

\section{Materials and Methods}

\section{Hip implant models}

Five of the most commonly used total hip prostheses (stem and respective cup, 0 Fig. 1 ) were immersed in agar gel (1\% agar) doped with Gadolinium contrast agent to mimic muscle tissue properties (at $1.5 \mathrm{~T} \mathrm{~T}_{1} \sim 1000 \mathrm{~ms}, \mathrm{~T}_{2} \sim 40 \mathrm{~ms}$ [18]). Special 3D-printed plastic bases were constructed to hold the material during gelification.

The stems are listed here with decreasing magnetic susceptibility:

- Stainless steel (magnetic susceptibility (x) 3520-67000 ppm [19])

1. Old Chanley prosthesis (Exeter stem ${ }^{\mathrm{TM}}$ Universal hip, Stryker Inc., Newbury, UK) is made of stainless steel for cemented fixation in total hip replacement. It is highly polished and it is an implant usually encountered in older patients. 

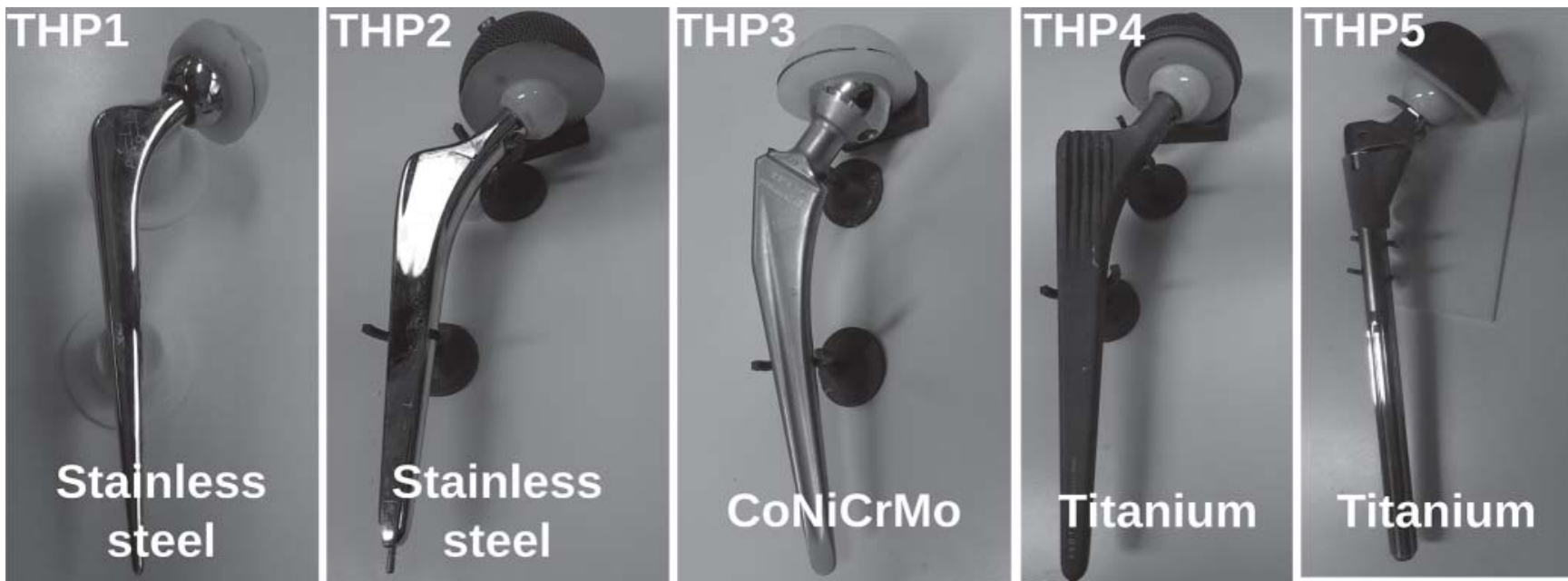

Fig. 1 Head and stem pictures of the five implants used in the present study. Prostheses were placed on plastic stands in order to be above the bottom of the container and surrounded by gel.

Abb. 1 Bilder des Kopfs und des Schafts der fünf Implantate, die in dieser Studie verwendet wurden. Die Prothesen wurden auf Sockeln aus Kunststoff über dem Boden des Behälters platziert und von Gel umgeben.

\begin{tabular}{|c|c|c|c|c|c|}
\hline & & $\mathrm{T}_{1-\mathrm{w}}$ SEMAC & & STIR SEMAC & \\
\hline & SES & scan time & & scan time ( & \\
\hline & & minimum & maximum & minimum & maximum \\
\hline 1 - Exeter & $10-17$ & 5.23 & 9.09 & 5.46 & 9.52 \\
\hline $2-M S-30$ & $6-16$ & 3.15 & 8.37 & 3.25 & 9.17 \\
\hline 3 - Müller & $7-14$ & 3.47 & 7.32 & 4.01 & 8.07 \\
\hline 4-Fitmore & $3-8$ & 1.38 & 4.19 & 1.40 & 4.36 \\
\hline 5-revision & $2-6$ & 1.06 & 3.15 & 1.35 & 3.25 \\
\hline reference & 23 & & & & \\
\hline
\end{tabular}

Table 1 Total scan times for the protocols used in the study for the individual materials.

Tab. 1 Messzeit der in der Studie für die einzelnen Materialien verwendeten Protokolle.

2. MS-30 stem $\left(\right.$ Zimmer $\left.^{\circledR}\right)$ is a stainless steel, highly polished, straight, three-dimensionally tapered, collarless implant for cemented fixation in total hip replacement. It is typically combined with a titanium acetabular cup.

- Cobalt-chromium alloys ( $X=900-1370$ ppm [19])

1. Müller type- CoNiCrMo alloy (Protasul ${ }^{\circledR}-10$ ) has a flat profile cap and is used with a cemented fixation.

- Titanium ( $\mathrm{X}=\sim 182 \mathrm{ppm}[19]$ )

1. A CLS ${ }^{\circledR}$ Spotorno ${ }^{\circledR}$ socket $\left(\right.$ Zimmer $\left.^{\circledR}\right)$ is combined with a fitmore stem (a grit-blasted titanium alloy with wedge shape). It is a cementless stem with a three-dimensional wedge shape and sharpened ribs in the proximal region. It is typically combined with a titanium acetabular cup.

2. S-Rom femoral component is a proximally modular revision prosthesis (DePuy Johnson \& Johnson). The S-Rom is a cementless femoral prosthesis consisting of a titanium stem. It is typically combined with a titanium acetabular cup.

\section{MR imaging}

MRI was performed on a $1.5 \mathrm{~T}$ clinical scanner (Magnetom Avanto, Siemens Medical Solutions, Erlangen, Germany). SEMAC (WARP TSE; Siemens Healthcare) was combined with high bandwidth radiofrequency pulses and increased readout bandwidth was used for the experiments. SEMAC also imbeds VAT for correction of in-plane distortion and additional 'slice' phase encoding steps (SES) in the slice direction for the correction of throughplane distortion. A six element body array was used for scanning.
Coronal planes were acquired (i.e., parallel to the longest axis of the stem) since they include both the stem and the head of the implant, giving a more complete image of the prosthesis surroundings and presenting higher clinical interest. Moreover, coronal slices require a large field-of-view and are thus more timedemanding.

Scout images were acquired with the SEMAC sequence with $4 \mathrm{SES}$, resolution $=2.2 \times 2.2 \times 3.0 \mathrm{~mm}^{3}$, FOV $=223 \times 280 \mathrm{~mm}^{2}$, flip angle $=140^{\circ}$, repetition time (TR) of $700 \mathrm{~ms}$, echo time (TE) of $4.7 \mathrm{~ms}$, parallel imaging factor (iPat) $=3$, scan time $=1.05 \mathrm{~min}$.

Coronal $\mathrm{T}_{1-\mathrm{w}}$ and fluid-sensitive STIR-SEMAC images were acquired with a variable number of SES. For the SEMAC $\mathrm{T}_{1-\mathrm{w}}$ protocol, the following parameters were used: $\mathrm{TR}=700 \mathrm{~ms}$, $\mathrm{TE}=4.9 \mathrm{~ms}$, flip angle $=130^{\circ}$, in-plane resolution $=0.88 \mathrm{~mm}$, slice thickness $=3.5 \mathrm{~mm}$, matrix size $=224 \times 280$, bandwidth per pixel $=781 \mathrm{~Hz}$, phase oversampling of $80 \%$, echo train length $(E T L)=23$, turbo factor $(T F)=7$. Parallel imaging (GRAPPA with iPAT $=3$ ) was used to speed up the acquisition for SEMAC. For STIR-SEMAC imaging, the parameters were: $\mathrm{TR}=5000 \mathrm{~ms}$, $\mathrm{TE}=35 \mathrm{~ms}$, flip angle $=150^{\circ}$, in-plane resolution $=0.94 \mathrm{~mm}$, slice thickness $=3 \mathrm{~mm}$, matrix size $=224 \times 302$. A number of SES from 2 to 23 was investigated depending on the susceptibility of every material (i. e., higher SES numbers for materials with stronger susceptibility, $\bullet$ Table 1 ). The range of SES was defined based on the results presented by Månson et al. [13]. For every implant an SES was selected as the minimum number and an optimal number of SES was defined from the analysis. The minimum number 
of SES was the minimum number of SES used for the measurements of a certain implant.

\section{Quantitative analysis}

In order to estimate the amount of artifact reduction as a function of SES, reference SEMAC images $\left(\mathrm{I}_{\text {ref }}\right)$ were acquired for every prosthesis material in the limit of a large number of SES (from scan time restrictions, 23 SES were considered as a reference SES). Then a series of SEMAC scans with various numbers of SES was performed $\left(\mathrm{I}_{\mathrm{SES}}\right)$. From this, relative signal difference images, $\mathrm{S}_{\mathrm{SES}}$, were calculated pixel-wise according to

$S_{\text {SES }}(x, y):=\frac{\left|I_{\text {SES }}(x, y)-I_{\text {ref }}(x, y)\right|}{I_{\text {ref }}(x, y)}$

The amount of hyperintense pixels $M$ in the difference image (i. e., pixels with intensity approximately 5 times above the noise) was chosen as an indicator for the quality of artifact suppression:

$\mathrm{M}:=\left\{(\mathrm{x}, \mathrm{y}) \in \mathrm{I} \mid \mathrm{S}_{\mathrm{SES}}>\right.$ thres $\}$

Finally, the volume of the distortion was calculated from

$\Delta$ Vol:=ord $(M) \times \operatorname{vol}_{\text {pixel }}$

The SES that was required to achieve a distortion below a certain threshold (i. e., $\Delta V$ Vol below $300 \mathrm{~mm}^{3}$ ) was defined as the optimal number of SES.

\section{Results}

$\nabla$

Coronal $\mathrm{T}_{1-\mathrm{w}}$ and STIR SEMAC images $\mathrm{I}_{\mathrm{SES}}$ were acquired for every implant, with a different initial SES depending on the material, until a stable artifact reduction level was achieved. The scan time increase versus the number of SES is depicted in $\bullet$ Fig. 2. Reference images $\mathrm{I}_{\mathrm{ref}}$ were also acquired from every phantom ( $\bullet$ Fig. 3). Initially, the $T_{1-w}$ SEMAC images and subsequently the STIR images were analyzed in order to determine a recommended number of SES for every material.
SEMAC: $\mathrm{T}_{1-\mathrm{w}}$ images

Distortion volumes for all five sets of prostheses as a function of SES are given in Fig. 4. As a result of the graphs, the optimal number of SES was established. The decreasing magnetic susceptibility of the materials (i.e., THP1 has the highest magnetic susceptibility and THP5 the lowest) is reflected in two observations: a) the shift of the curves towards the left, b) the lower optimal number of SES (i. e., SES required for decrease of the distortion volume below the threshold). The comparison of different protocols showed that for every material there is a point at which the volume of distortion decreases below the threshold of $300 \mathrm{~mm}^{3}$ (i. e., reflecting that the distortion in a certain image becomes the same as in the reference image) and the use of more slice encoding steps brings no considerable reduction of the signal void.

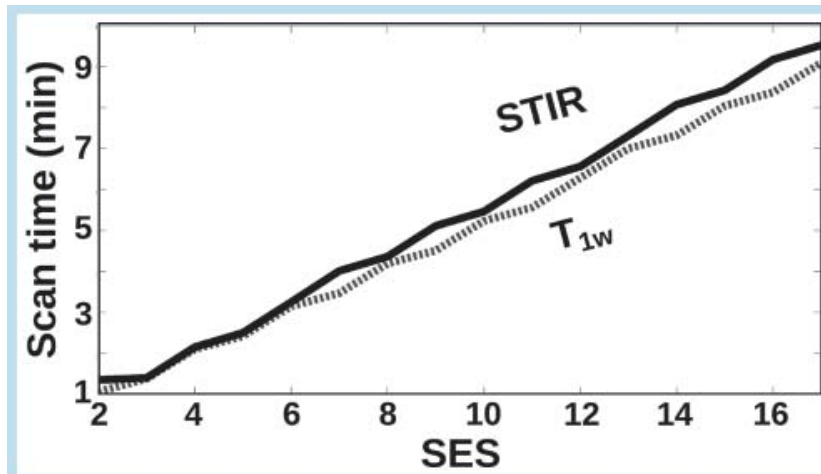

Fig. 2 Total scan time of the $\mathrm{T}_{1-\mathrm{w}}$ (dotted) and STIR (solid) SEMAC protocols used in the study as a function of the number of slice encoding steps (SES).

Abb. 2 Messzeit der in der Studie verwendeten $\mathrm{T}_{1-\mathrm{w}}$ (gepunktete Linie) und STIR (durchgezogene Linie) SEMAC-Protokolle als Funktion der Anzahl an Schichtkodierungsschritten (SES).

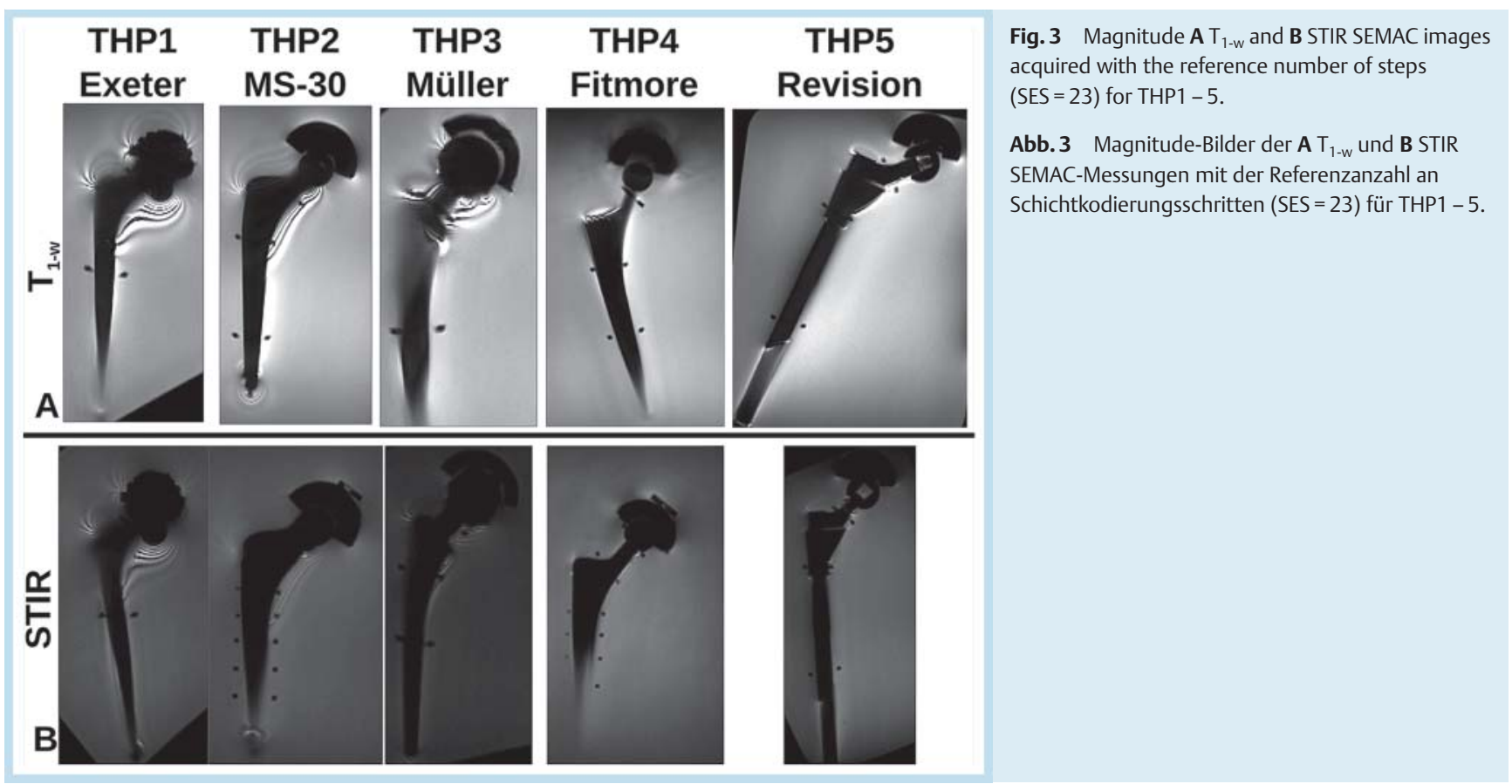




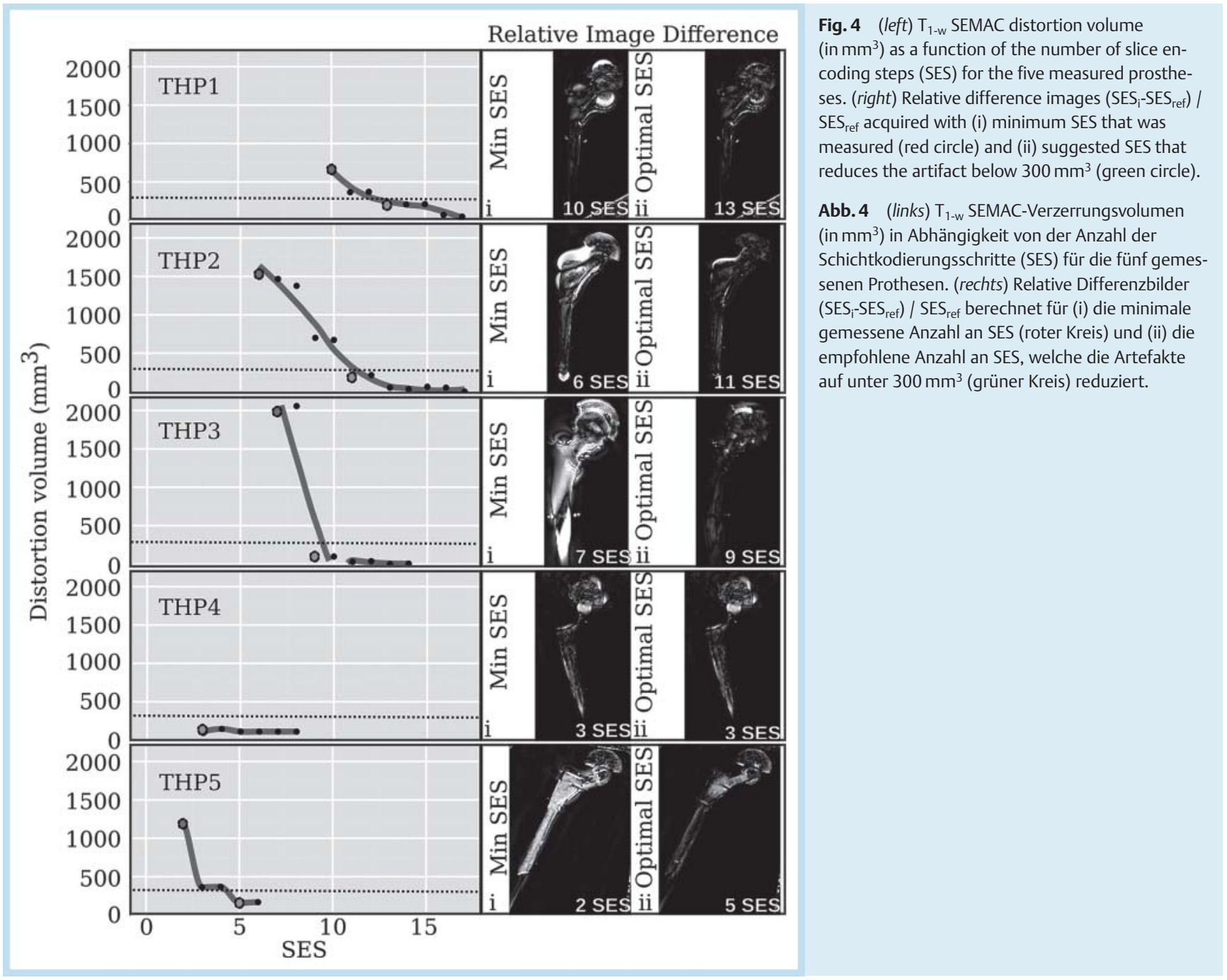

a) Stainless steel-based implants (THP1 \& THP2)

For THP1 (Exeter), which shows the most prominent susceptibility artifacts, the volume of the distortion is decreased below $300 \mathrm{~mm}^{3}$ for SES above 13. For THP2, which is also made of stainless steel, the volume decreases below the threshold for a lower SES [11] and the curve is slightly shifted to the left. Therefore, it is concluded that depending on the alloy, stainless steel prostheses can have a slightly different behavior in the presence of a magnetic field. A number of SES of at least 11 and preferably higher than 13 is recommended in both cases. The respective $\mathrm{T}_{1-\mathrm{w}}$ images for the minimum and optimal number of SES are presented on $\odot$ Fig. 4. For a very small number of SES (10 for THP1 and 6 THP2, $\odot$ Fig. 4), there is important signal distortion that is mainly centered in the area of the neck of the stem. For the optimal number of SES (i. e., 13 for THP1 and 11 for THP2), it can be observed that in both cases the relative difference images have minimum hyperintense regions and therefore above this level there is only a very small gain using a higher number of SES.

\section{b) Cobalt-chromium-based implant (THP3)}

In this case the artifact reduces considerably in images acquired with a number of SES greater than or equal to 9 ( $\bullet$ Fig. 4). For an SES number above 9 , the curve is practically flat and the distortion is almost identical to the distortion in the reference image. From a qualitative evaluation of the images, it can be seen that the relative difference image has only a very small hyperintense region close to the top of the stem and there is no considerable gain in increasing the number of SES above this limit.

\section{c) Titanium-based implants (THP4 \& THP5)}

For THP4 and THP5 (titanium stems), $\mathrm{T}_{1-\mathrm{w}}$ SEMAC images were acquired with a lower number of steps. For the THP4 and THP5, both from a quantitative and qualitative evaluation of the images ( $\odot$ Fig. 4), it can be observed that the geometric distortion is already very restricted, when using 3 and 5 SES, respectively. Therefore, for titanium-based alloys it can be considered that it is not profitable to acquire images with more than 5 SES.

\section{SEMAC: STIR images}

The results are almost identical ( $\bullet$ Fig. 5) for the STIR SEMAC images with very small differences. The curves show similar steepness and limits of SES that effectively minimize the volume of the distortion. The volume of distortion is higher in the $\mathrm{T}_{1-\mathrm{w}}$ images only for THP2. However, the steepness of the curves is comparable and in both cases the distortion volume for a number of SES above 10 decreases below $300 \mathrm{~mm}^{3}$. The recommendations for SES therefore can be considered the same. 


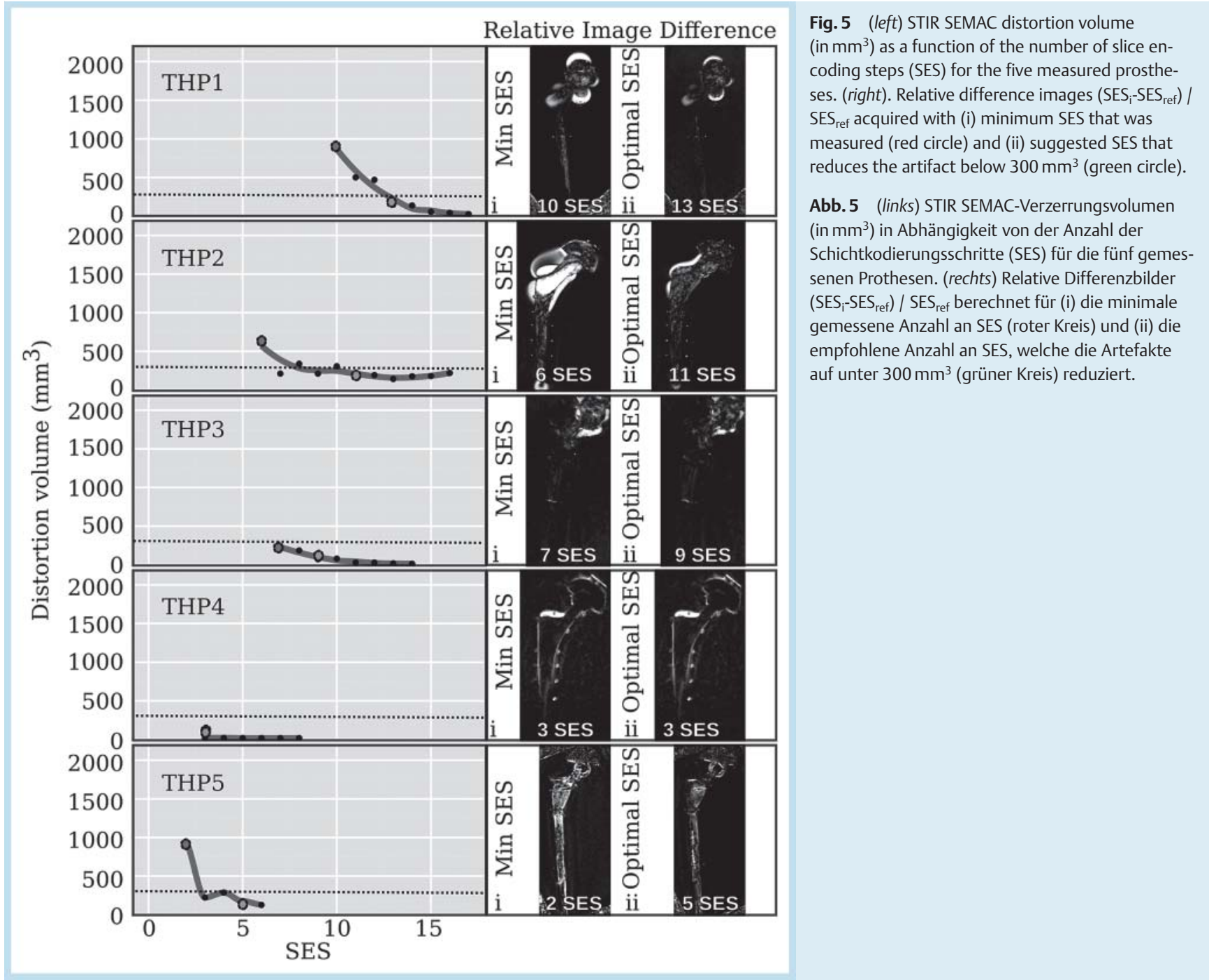

\section{Discussion}

$\nabla$

THA is performed as a treatment for degenerative joint disease with many possible complications, such as loosening and infection [1]. MRI is an important tool for the diagnosis of these complications, but suffers from pronounced magnetic susceptibility artifacts in the presence of metal. Recent advances in sequence development, however, can provide diagnostic images even in the presence of hip prostheses $[2,6,7,9,11,20]$. These techniques allow improved image quality by a considerable reduction of the artifact volume at the expense of prolonged scan times. The range of materials for hip prostheses is very wide, depending also on the age of the patient. As a result, the implant material is usually unknown, the imaging protocols are not optimized based on the material and clinical studies do not differentiate results based on prostheses models.

Recently, there has been an increased interest in studying the influence of implant material on the performance of an artifact reduction sequence $[13,14,16,17]$. Månson et al. presented a method for evaluating the performance of different techniques using SEMAC, VAT and $\mathrm{T}_{1-\mathrm{w}}$ TSE sequences with different prostheses. They studied the performance of TSE, VAT and SEMAC sequences with three different prostheses. We extended the results of this study to include an evaluation of a continuous range of SES and two different types of contrast for the SEMAC protocols $\left(\mathrm{T}_{1-\mathrm{w}}\right.$ and STIR). The contrast type did not have a pronounced effect on the optimal number of SES. In addition, in this study five different implants were investigated.

The materials of the implants could be categorized into some general groups (i. e., titanium-based, stainless steel-based, etc.). Based on the extent of image distortions for the five examined implants, three different groups of implant categories were identified:

- Strong susceptibility materials (e.g. stainless steel): They create very strong susceptibility artifacts and can often be encountered in older patients. On SEMAC images, these implants show a considerable artifact around the area of the neck of the stem, which extends far beyond the vicinity of the implant, and it is recommended to use an SES number equal to 13.

- Medium susceptibility materials (e.g. CoNiCrMo): In the SEMAC images, geometric distortion is effectively minimized for an SES number equal to 9 .

- Low magnetic susceptibility (e.g. titanium): They are more modern or often replacement prostheses. These implants can in principle already be visualized with optimized high bandwidth TSE protocols and it is sufficient to use 5 SES.

Although no external volume reference such as X-ray [13] was used at this point, a comparison with a protocol using a very high SES number gave a clear evaluation of the protocol efficien- 
cy. At present, only in vitro experiments were performed and our results need to be validated in vivo. Another limitation might arise from the image orientation. Here, we focused on coronal protocols (i.e., parallel to the longest axis of the stem), which is the plane with the highest clinical interest. Nevertheless, it would be important to confirm whether the same guidelines are true for different image planes in order to establish comprehensive clinical recommendations.

\section{Conclusion}

In a standard clinical setting the clinician may not know the exact implant of a patient at the time of examination. However, optimization of the metal artifact reduction protocol based on the implant material can lead to a more time-efficient scan with better image quality. In this study, we established scan time recommended SES for five different total hip prostheses.

\section{Clinical Relevance of the Study}

- The results could serve as a guideline in order to trade acquisition time against diagnostic image quality depending on the material that is present.

- A minimum number of slice encoding steps (5 SES) allows adequate image quality of titanium prostheses.

- For non-titanium-based implants that cause medium distortion such as cobalt-chromium-based alloys, more SES are needed (9 SES) to minimize metal artifacts.

- For older prostheses made out of stronger metals, a higher number of SES (13 SES) is beneficial in order to effectively reduce the area of the artifacts.

\section{Acknowledgements}

$\nabla$

We thank Siemens Healthcare for providing the prototype SEMAC sequence.

We thank Francesco Santini for his support regarding the design of the 3D-printed phantom bases and Rahel Heule proofreading the manuscript.

\section{References}

1 Kurtz S, Mowat F, Ong $K$ et al. Prevalence of primary and revision total hip and knee arthroplasty in the United States from 1990 through 2002. J Bone Joint Surg Am 2005; 87: 1487-1497

2 Koch KM, Hargreaves BA, Pauly KB et al. Magnetic resonance imaging near metal implants. Journal of Magnetic Resonance Imaging 2010; 32: $773-787$

3 Cahir JG, Toms AP, Marshall TJ et al. CT and MRI of hip arthroplasty. Clinical Radiology 2007; 62: 1163-1171

4 Toms AP, Smith-Bateman C, Malcolm PN et al. Optimization of metal artefact reduction (MAR) sequences for MRI of total hip prostheses. Clinical Radiology 2010; 65: 447-452

5 Hargreaves BA, Worters PW, Pauly KB et al. Metal-induced artifacts in MRI. Am J Roentgenol 2011; 197: 547-555

6 Cho ZH, Kim DJ, Kim YK. Total inhomogeneity correction including chemical shifts and susceptibility by view angle tilting. Med Phys 1988; 15: 7-11

7 Koch K, Lorbiecki J, Hinks R et al. A multispectral three-dimensional acquisition technique for imaging near metal implants. Magn Reson Med 2009; 61: 381 - 390

8 Artz NS, Hernando D, Taviani $V$ et al. Spectrally resolved fully phaseencoded three-dimensional fast spin-echo imaging. Magn Reson Med 2014; 71: 681-690

9 Lu W, Pauly KB, Gold GE et al. SEMAC: Slice Encoding for Metal Artifact Correction in MRI. Magn Reson Med 2009; 62: 66-76

10 Sutter R, Ulbrich EJ,Jellus V et al. Reduction of metal artifacts in patients with total hip arthroplasty with slice-encoding metal artifact correction and view-angle tilting MR imaging. Radiology 2012; 265: $204-$ 214

11 Chen CA, Chen W, Goodman SB et al. New MR Imaging Methods for Metallic Implants in the Knee: Artifact Correction and Clinical Impact. Journal of magnetic resonance imaging: JMRI 2011; 33: 1121-1127

12 Sutter R, Hodek R, Fucentese SF et al. Total knee arthroplasty MRI featuring slice-encoding for metal artifact correction: reduction of artifacts for STIR and proton density-weighted sequences. Am J Roentgenol 2013; 201: 1315-1324

13 Månsson S, Müller GM, Wellman F et al. Phantom based qualitative and quantitative evaluation of artifacts in MR images of metallic hip prostheses. Physica Medica 2015; 31: 173-178

14 Müller GM, Lundin B, von Schewelov Tet al. Evaluation of metal artifacts in clinical MR images of patients with total hip arthroplasty using different metal artifact-reducing sequences. Skeletal Radiol 2015; 44: $353-359$

15 Ai T, Padua A, Goerner $F$ et al. SEMAC-VAT and MSVAT-SPACE sequence strategies for metal artifact reduction in $1.5 \mathrm{~T}$ magnetic resonance imaging. Investigative radiology 2012; 47: $267-276$

16 Koff MF, Shah P, Koch KM et al. Quantifying image distortion of orthopedic materials in magnetic resonance imaging. J Magn Reson Imaging 2013; 38: 610-618

17 Panfili E, Pierdicca L, Salvolini L et al. Magnetic resonance imaging (MRI) artefacts in hip prostheses: a comparison of different prosthetic compositions. Radiol Med 2013; 119: 113-120

18 Stanisz GJ, Odrobina EE, Pun J et al. T1, T2 relaxation and magnetization transfer in tissue at 3T. Magnetic Resonance in Medicine 2005; 54: $507-512$

19 Chang EY, Bae WC, Chung CB. Imaging the Knee in the Setting of Metal Hardware. Magnetic Resonance Imaging Clinics of North America 2014; 22: 765-786

20 Agten CA, Sutter R, Pfirrmann CWA. CT and MRI of hip arthroplasty. Radiologe 2014; 54: 715-726 